\title{
LAMINAR BOUNDARY LAYER FLOW OVER A CONE WITH SUCTION OR INJECTION*
}

\author{
BY \\ TAKASHI WATANABE \\ Iwate University, Morioka, Japan
}

\begin{abstract}
The effect of uniform suction or injection on the flow of an incompressible laminar boundary layer over a cone was theoretically investigated. The boundary layer equations along a cone are transformed into nonsimilar ones, and the numerical calculations of the resulting equations are performed by the difference-differential method for various values of the suction/injection parameter. The neutral staijility curves are then presented for values of the cone angle and the suction/injection parameter. The results are given for the velocity profiles, coefficient of skin friction, displacement thickness, and the critical Reynolds numbers.
\end{abstract}

1. Introduction. The problem of three-dimensional boundary layer flow past an axisymmetric body is of fundamental importance in fluid mechanics. The behavior of a boundary layer over a cone is one of the simplest types of axisymmetric flow. The fluid flow and the heat and mass transfer over a cone rotating in a still medium have been studied by many investigators [1-5]. However, studies of the stability and the transition of boundary layer over a cone have not been reported in detail except for the experimental and theoretical investigation done by Hassler [6] and Kobayashi [7].

The purpose of the present paper is to solve a nonsimilar boundary layer flow with uniform suction or injection and to study the theory for the linear stability analysis of the effect of uniform suction or injection on the flow over a cone. The nonsimilar boundary layer equation was calculated by the difference-differential method after transforming it to an equivalent two-dimensional problem by Mangler's transformation. Numerical results were obtained for the velocity profiles, displacement thickness, and the coefficient of skin friction for various values of suction/injection parameter. The effect of the suction or injection on the stability of boundary layer over a cone was further considered. The neutral stability curves and the critical Reynolds numbers were given for various values of suction/injection parameter.

2. Laminar boundary layer along a cone. A laminar boundary layer flow with uniform suction or injection of an incompressible fluid along a cone is considered. The coordinates and the velocities of the axially symmetrical problem are first transformed

${ }^{*}$ Received March 12, 1987.

(C) 1988 Brown University 
into those of the equivalent two-dimensional problem by the Mangler's transformation. The equation of motion and of continuity for the boundary layer of an incompressible fluid can be written after transformation as follows:

$$
\begin{gathered}
u \frac{\partial u}{\partial x}+v \frac{\partial u}{\partial y}=\nu\left(\frac{\partial^{2} u}{\partial y^{2}}\right)+U_{e} \frac{d U_{e}}{d x}, \\
\frac{\partial u}{\partial x}+\frac{\partial v}{\partial y}=0
\end{gathered}
$$

with the boundary conditions being

$$
\begin{gathered}
y=0: u=0, \quad v=v_{0}, \\
y \rightarrow \infty: u=U_{e}=a x^{m / 3},
\end{gathered}
$$

where $x$ and $y$ are taken respectively as the coordinates parallel and normal to the wall, $u$ and $v$ denoting the components of the fluid velocity in the $x$ and $y$ direction, respectively. Next, the following transformation making use of the stream function will be used:

$$
\begin{aligned}
& u=\frac{\partial \psi}{\partial y}, \quad v=-\frac{\partial \psi}{\partial x}, \quad \psi=\left(\frac{6}{m+3} \nu x U_{e}\right)^{1 / 2} f(x, \eta), \\
& \eta=y\left(\frac{m+3}{6} \frac{U_{e}}{\nu x}\right)^{1 / 2} .
\end{aligned}
$$

The component of the velocity can be expressed as

$$
\begin{aligned}
& u=U_{e} \frac{\partial f}{\partial \eta}, \\
& v=-\sqrt{\frac{6}{m+3} \frac{\nu U_{e}}{x}}\left(\frac{1}{2} f+\frac{1}{2} \frac{x}{U_{e}} \frac{d U_{e}}{d x} f+x \frac{\partial f}{\partial \eta} \frac{\partial \eta}{\partial x}+x \frac{\partial f}{\partial x}\right) .
\end{aligned}
$$

Introducing Eqs. (5) and (6) in Eqs. (1) and (2), the governing differential equation becomes

$$
\begin{aligned}
\frac{\partial^{3} f}{\partial \eta^{3}}+ & f \frac{\partial^{2} f}{\partial \eta^{2}}+\frac{2 m}{m+3}\left\{1-\left(\frac{\partial f}{\partial \eta}\right)^{2}\right\} \\
& +\frac{6}{m+3} x\left(\frac{\partial f}{\partial x} \frac{\partial^{2} f}{\partial \eta^{2}}-\frac{\partial^{2} f}{\partial x \partial \eta} \frac{\partial f}{\partial \eta}\right)=0
\end{aligned}
$$

and the boundary conditions

$$
\begin{aligned}
& \eta=0: \frac{\partial f}{\partial \eta}=0, \quad \frac{1}{2} f+\frac{1}{2} \frac{x}{U_{e}} \frac{d U_{e}}{d x} f+x \frac{\partial f}{\partial x}=k \sqrt{x}, \\
& \eta \rightarrow \infty: \frac{\partial f}{\partial \eta}=1,
\end{aligned}
$$

where $k \sqrt{x}$ is the parameter of suction or injection which is defined by

$$
k \sqrt{x}=-v_{0} \sqrt{\frac{m+3}{6} \frac{x}{\nu U_{e}}} .
$$

$U_{e}$ is the flow velocity at the outer edge of the boundary layer, which is given as $U_{e}(x)=a x^{\frac{m}{3}} . \quad v_{0}$ is the velocity of suction $\left(v_{0}<0\right)$ or injection $\left(v_{0}>0\right)$. The 
parameter $m$ depends solely on the cone half angle $\Omega$. The relation between $m$ and $\Omega$ was shown by Whitehead and Canetti [5], and the tabulated values of $m$ and $\Omega$ were given by Hess-Faulkner [8]. The partial differential equation (7) is approximated by a system of ordinary differential equations when replacing the $x$ derivative by finite difference. After substituting $X=k \sqrt{x}$, Eq. (7) is rewritten as

$$
\frac{\partial^{3} f}{\partial \eta^{3}}+\left(f+\frac{3}{m+3} X \frac{\partial f}{\partial X}\right) \frac{\partial^{2} f}{\partial \eta^{2}}-\frac{3}{m+3} X \frac{\partial^{2} f}{\partial X \partial \eta} \frac{\partial f}{\partial \eta}+\frac{2 m}{m+3}\left\{1-\left(\frac{\partial f}{\partial \eta}\right)^{2}\right\}=0
$$

The boundary conditions ( 8 ) become

$$
\begin{gathered}
\eta=0: \frac{\partial f}{\partial \eta}=0, \quad \frac{1}{3}(m+3) f+X \frac{\partial f}{\partial X}=X, \\
\eta \rightarrow \infty: \frac{\partial f}{\partial \eta}=1 .
\end{gathered}
$$

By using a four-point formula of Gregory-Newton, as an example, the derivative for $X$ at $X=X_{i}=i h(i=0,1,2, \ldots)$, where $h$ is a constant interval, can be approximated. Equation (10) becomes the following ordinary differential equation:

$$
\begin{aligned}
\frac{d^{3} f_{i}}{d \eta^{3}} & +\left\{f_{i}+\frac{3}{m+3} \frac{i}{6}\left(11 f_{i}-18 f_{i-1}+9 f_{i-2}-2 f_{i-3}\right)\right\} \frac{d^{2} f_{i}}{d \eta^{2}} \\
& -\frac{3}{m+3} \frac{i}{6}\left(11 \frac{d f_{i}}{d \eta}-18 \frac{d f_{i-1}}{d \eta}+9 \frac{d f_{i-2}}{d \eta}-2 \frac{d f_{i-3}}{d \eta}\right) \frac{d f_{i}}{d \eta} \\
& +\frac{2 m}{m+3}\left\{1-\left(\frac{d f_{i}}{d \eta}\right)^{2}\right\}=0 .
\end{aligned}
$$

The boundary conditions (11) are then replaced by

$$
\begin{gathered}
\eta=0: \frac{d f_{i}}{d \eta}=0, \quad f_{i}=i h, \\
\eta \rightarrow \infty: \frac{d f_{i}}{d \eta}=1 .
\end{gathered}
$$

The solution of Eq. (12) is expressed as an integral at the $i$ th station $X=i h$,

$$
\begin{aligned}
\frac{d f_{i}}{d \eta}=\left\{1-\int_{0}^{\infty} E(\eta)\right. & \left.\int_{0}^{\eta} \frac{R(\eta)}{E(\eta)} d \eta d \eta\right\} \frac{G(\eta)}{G(\infty)} \\
& +\int_{0}^{\eta} E(\eta) \int_{0}^{\eta} \frac{R(\eta)}{E(\eta)} d \eta d \eta \\
f_{i} & = \pm i h+\int_{0}^{\eta} \frac{d f_{i}}{d \eta} d \eta
\end{aligned}
$$


where

$$
\begin{aligned}
E(\eta)= & \exp \left[\int_{0}^{\eta}\left\{-f_{i}-\frac{3}{m+3} \frac{i}{6}\left(11 f_{i}-18 f_{i-1}+9 f_{i-2}-2 f_{i-3}\right)\right\} d \eta\right], \\
R(\eta)= & \frac{3}{m+3} \frac{i}{6}\left(11 \frac{d f_{i}}{d \eta}-18 \frac{d f_{i-1}}{d \eta}+9 \frac{d f_{i-2}}{d \eta}-2 \frac{d f_{i-3}}{d \eta}\right) \frac{d f_{i}}{d \eta} \\
& -\frac{2 m}{m+3}\left\{1-\left(\frac{d f_{i}}{d \eta}\right)^{2}\right\}, \\
G(\eta)= & \int_{0}^{\eta} E(\eta) d \eta .
\end{aligned}
$$

The plus and minus sign, in Eq. (15), refers to the suction and injection respectively. Equations (14) and (15) involve $f(\eta)$ and $d f / d \eta$ at the station $X=X_{i-1}, X_{i-2}$, and $X_{i-3}$. When the quantities at all previous stations are known, the solutions at the $X=X_{i}$ can be determined. The Falkner-Skan solution can easily be recognized in the initial function $f_{0}(\eta)$ at $X=0$. The quantities $f_{1}(\eta)$ and $f_{2}(\eta)$ at $X=X_{1}$ and $X=X_{2}$ can be obtained by introducing equations similar to Eq. (12), in which the derivatives of $f(\eta)$ with respect to $X$ are replaced by two-point or three-point formulas instead of a four-point formula. The integral equations (14) and (15) were solved by iterative numerical procedures to a positive or negative $X$ direction according to the sign of $k \sqrt{x}$. The convergence of the iteration was kept within $5 \times 10^{-7}$ for values of $d^{2} f / d \eta^{2}$ at $\eta=0$. The velocity profiles $U\left(=u / U_{e}\right)$ in the boundary layer are shown in Fig. 1 for various values of $\Omega$. Figures 2,3 , and 4 show the velocity profiles for $\Omega=15^{\circ}, 30^{\circ}$, and $60^{\circ}$, respectively, as a function of the suction/injection parameter $k \sqrt{x}$. The velocity profiles become flat as the cone angle $\Omega$ and the suction/injection parameter $k \sqrt{x}$ are increased. Figure 5 shows the relation between the displacement thickness $\delta^{*}$ and the suction/injection parameter $k \sqrt{x}$ for various values of the cone angle $\Omega . \delta^{*}$ is calculated from the following equation:

$$
\delta^{*}=\int_{0}^{\infty}\left(1-\frac{u}{U_{e}}\right) d y=\sqrt{\frac{6}{m+3} \frac{\nu x}{U_{e}}} \int_{0}^{\infty}\left(1-\frac{d f}{d \eta}\right) d \eta .
$$

As it can be seen in Fig. 5, the displacement thickness $\delta^{*}$ decreases with increasing cone angle $\Omega$ and suction/injection parameter $k \sqrt{x}$. The coefficient of skin friction $C_{f}$ is shown in Fig. 6 against $k \sqrt{x}$, where the coefficient $C_{f}$ is defined by

$$
C_{f}=2 \mu\left(\frac{\partial u}{\partial y}\right)_{y=0}\left(\rho U_{e}^{2}\right)^{-1}=2 \sqrt{\frac{m+3}{6} \frac{\nu}{U_{e} x}}\left(\frac{d^{2} f}{d \eta^{2}}\right)_{\eta=0} .
$$

By observation, the coefficient of skin friction $C_{f}$, in Fig. 6 , is seen to increase for all cone angles as $k \sqrt{x}$ is increased.

3. Stability of laminar boundary layer over a cone. The effect of uniform suction or injection on the stability of the boundary layer mentioned above is considered. In this analysis, the Orr-Sommerfeld equation is used as the perturbation equation, which is expressed as follows:

$$
(U-c)\left(\frac{d^{2} \phi}{d \eta^{2}}-\alpha^{2} \phi\right)-\phi \frac{d^{2} U}{d \eta^{2}}=-\frac{i}{\alpha R_{e}}\left(\frac{d^{4} \phi}{d \eta^{4}}-2 \alpha^{2} \frac{d^{2} \phi}{d \eta^{2}}-\alpha^{4} \phi\right) .
$$




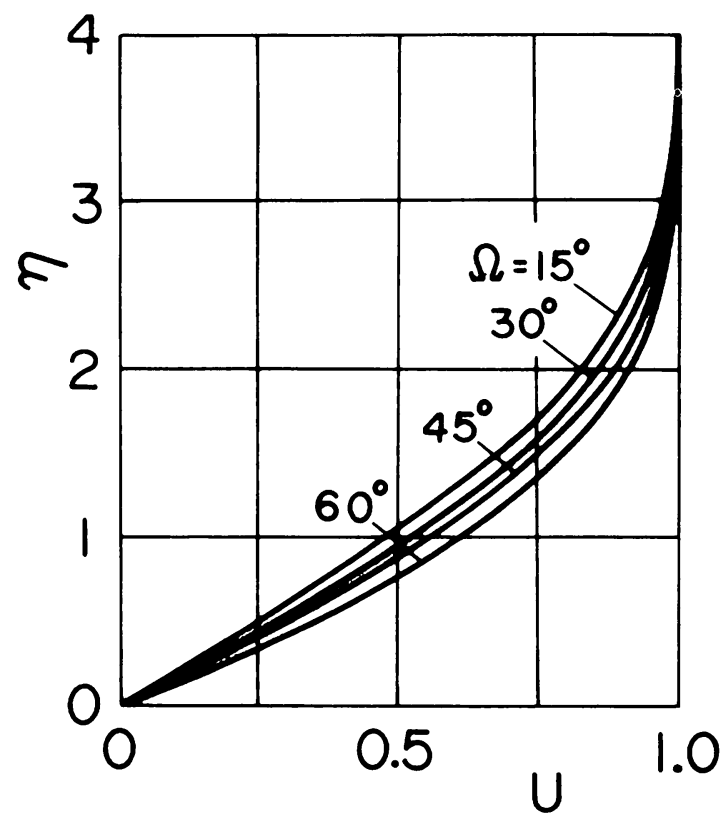

Fig. 1. Velocity profiles $(k \sqrt{x}=0)$.

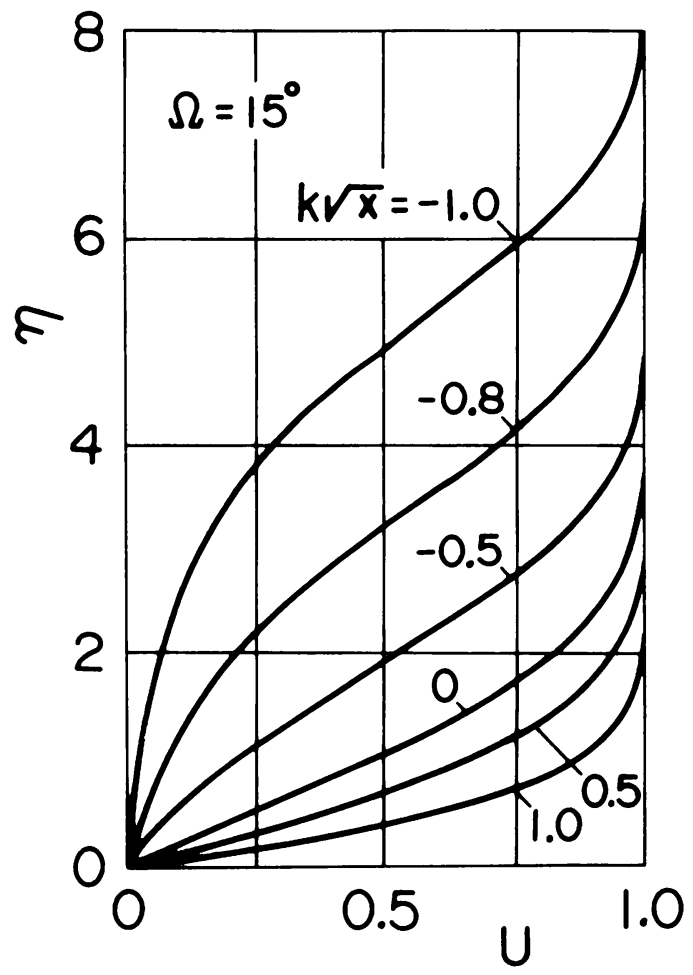

FiG. 2. Velocity profiles $\left(\Omega=15^{\circ}\right)$. 


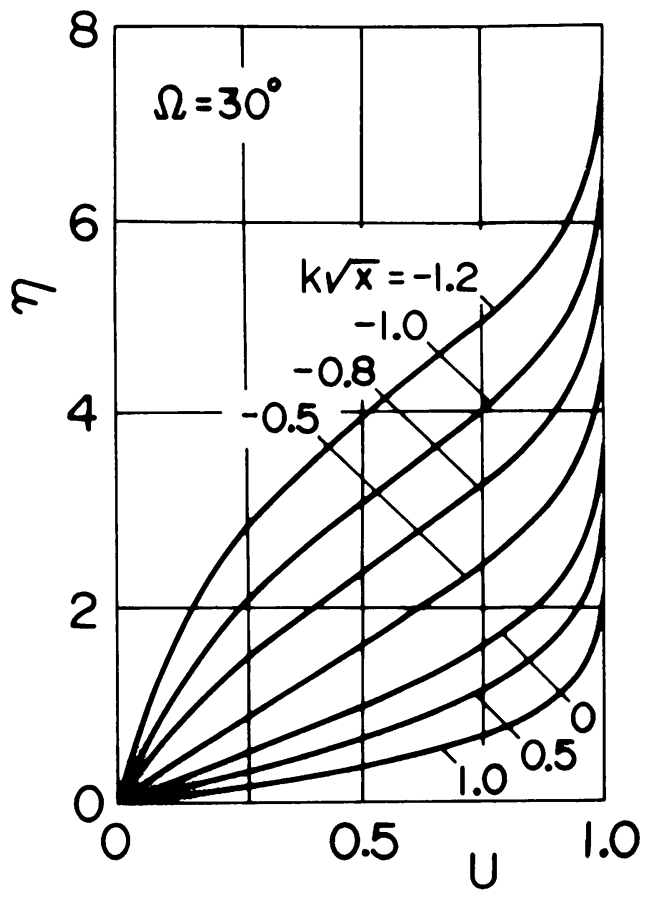

FIG. 3. Velocity profiles $\left(\Omega=30^{\circ}\right)$.

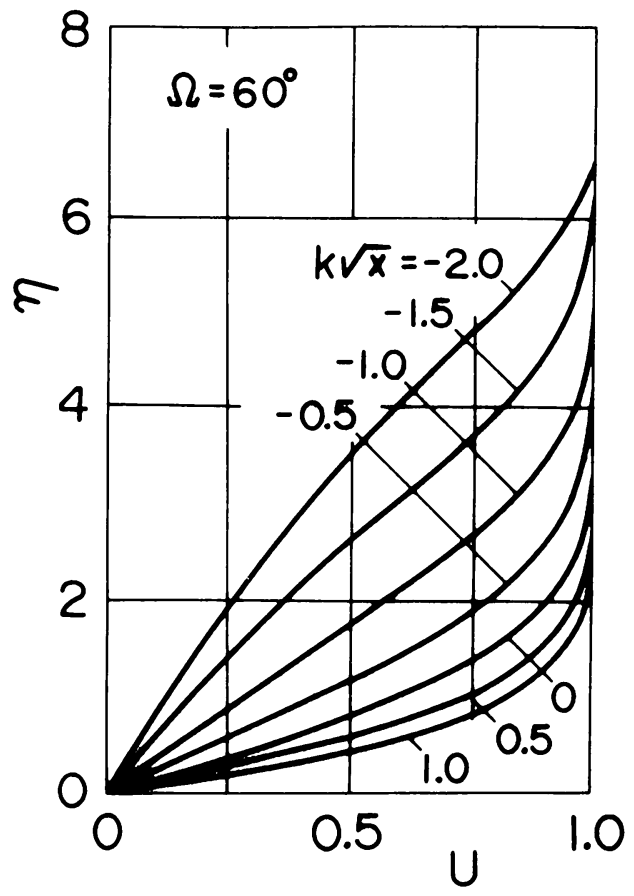

Fig. 4. Velocity profiles $\left(\Omega=60^{\circ}\right)$. 
This means that the principal effect is the modification of the velocity distribution $U$ in the present problem. The neutral stability curves are calculated for the velocity profiles shown in Figs. 2, 3, and 4 by Lin's method of analysis [9]. The results are shown in Figs. 7, 8, and 9 for the cone half angle $\Omega=15^{\circ}, 30^{\circ}, 60^{\circ}$, respectively. The nondimensional wave number of disturbances $\alpha^{*}$ is plotted against the Reynolds number $R_{e}^{*} \cdot \alpha^{*}$ and $R_{e}^{*}$ are given as $\alpha^{*}=\alpha \delta^{*}$ and $R_{e}^{*}=U_{e} \delta^{*} / \nu$, as a function of the suction/injection parameter $k \sqrt{x}$. Figure 10 shows the relation between the critical Reynolds number $R_{e c}^{*}$ and the suction/injection parameter $k \sqrt{x}$. It is clear from these figures that the critical Reynolds number $R_{e c}^{*}$ increases as the cone angle $\Omega$ and the suction/injection parameter $k \sqrt{x}$ are increased, that is, the flow is stabilized.

4. Conclusions. The characteristics of the boundary layer over a cone for the case of uniform suction or injection were made quantitatively clear. The theoretical analysis on the growth of instabilities of the boundary layer were further carried out. The results are summarized as follows.

1. The velocity profiles, the displacement thickness, and the skin friction were given, which made the calculation of the neutral stability curves become possible. As the cone angle and the suction/injection parameter are increased, the coefficient of skin friction increases and the boundary layer displacement thickness decreases.

2. The curves of neutral stability were obtained for various values of the cone angle and suction/injection parameter. As the cone angle and the suction/injection parameter are increased, the critical Reynolds number increases, as the result of which, the boundary layer flow along a cone becomes stable.

Acknowledgment. The author wishes to express his hearty thanks to Prof. R. Kobayashi, Tohoku University, for guidance and encouragement. 


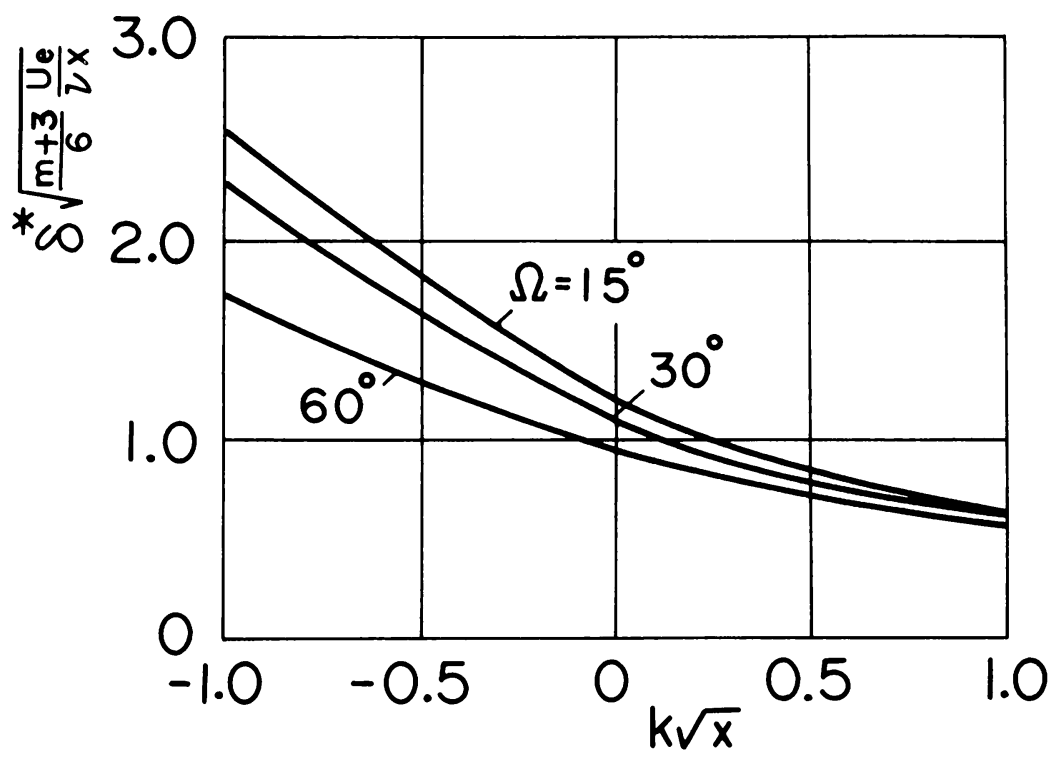

FIG. 5. Displacement thickness $\delta^{*}$ in relation to the suction/injection parameter $k \sqrt{x}$.

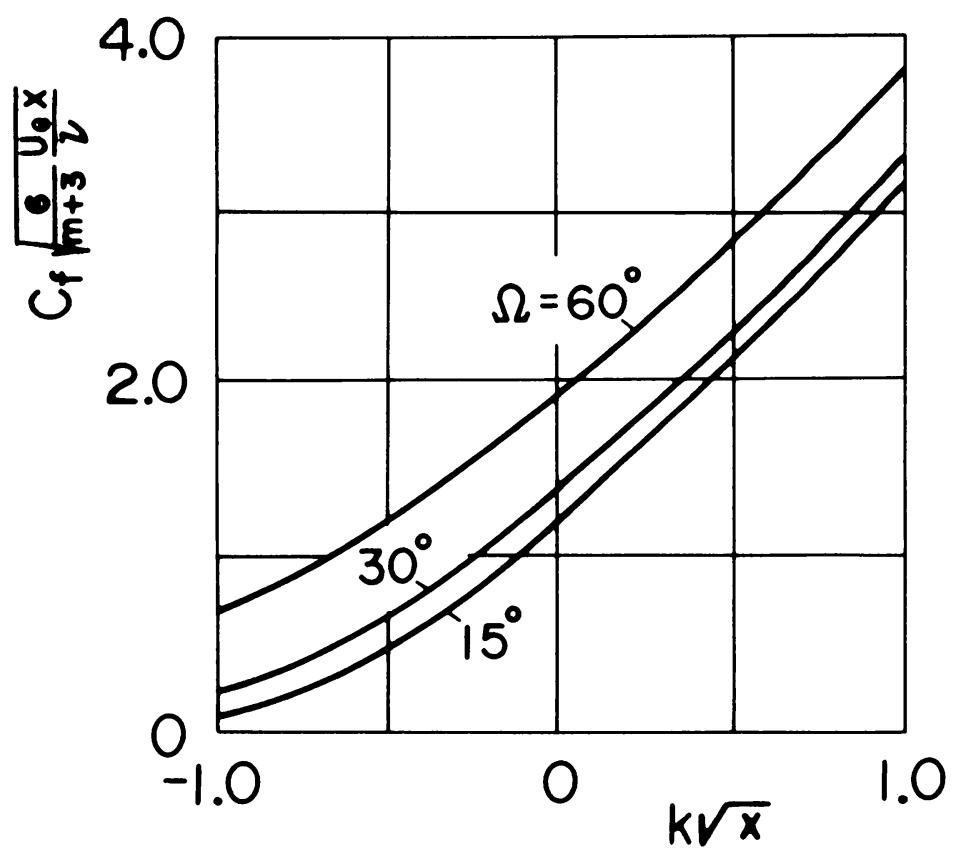

FIg. 6. Coefficient of skin friction $C_{f}$ against the suction/injection parameter $k \sqrt{x}$. 


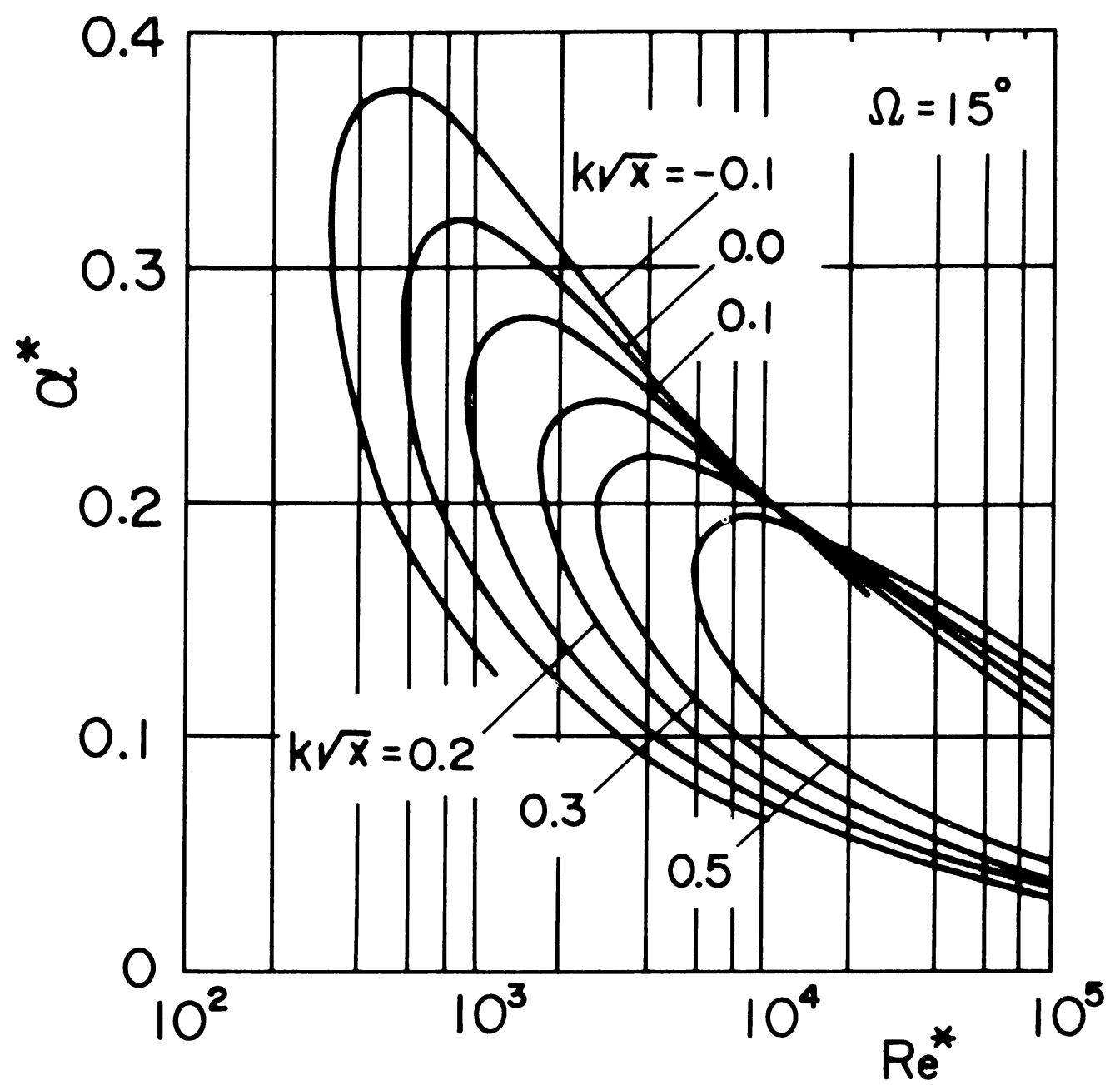

Fig. 7. Neutral stability curves $\left(\Omega=15^{\circ}\right)$. 


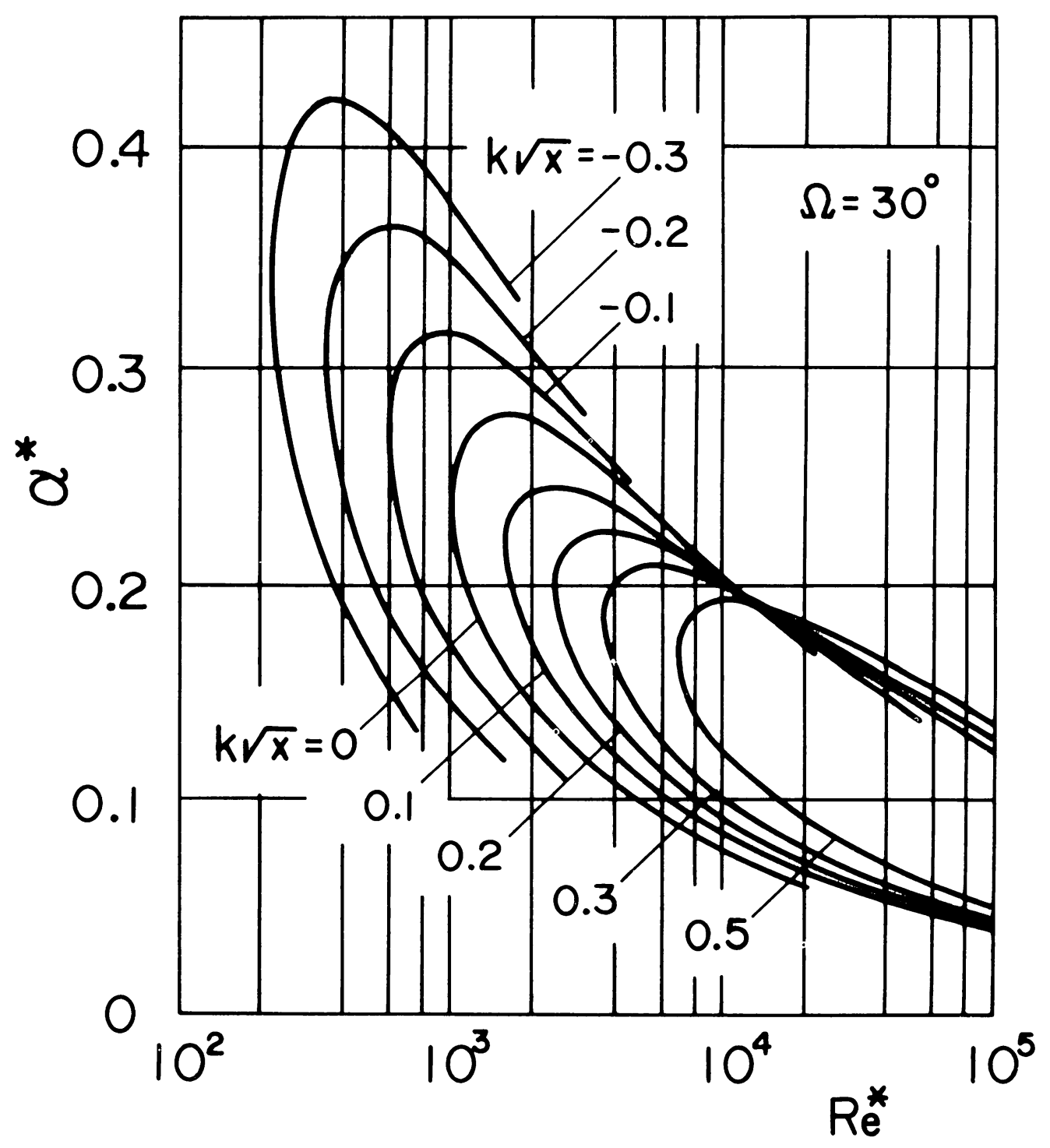

FIG. 8 . Neutral stability curves $\left(\Omega=30^{\circ}\right)$. 


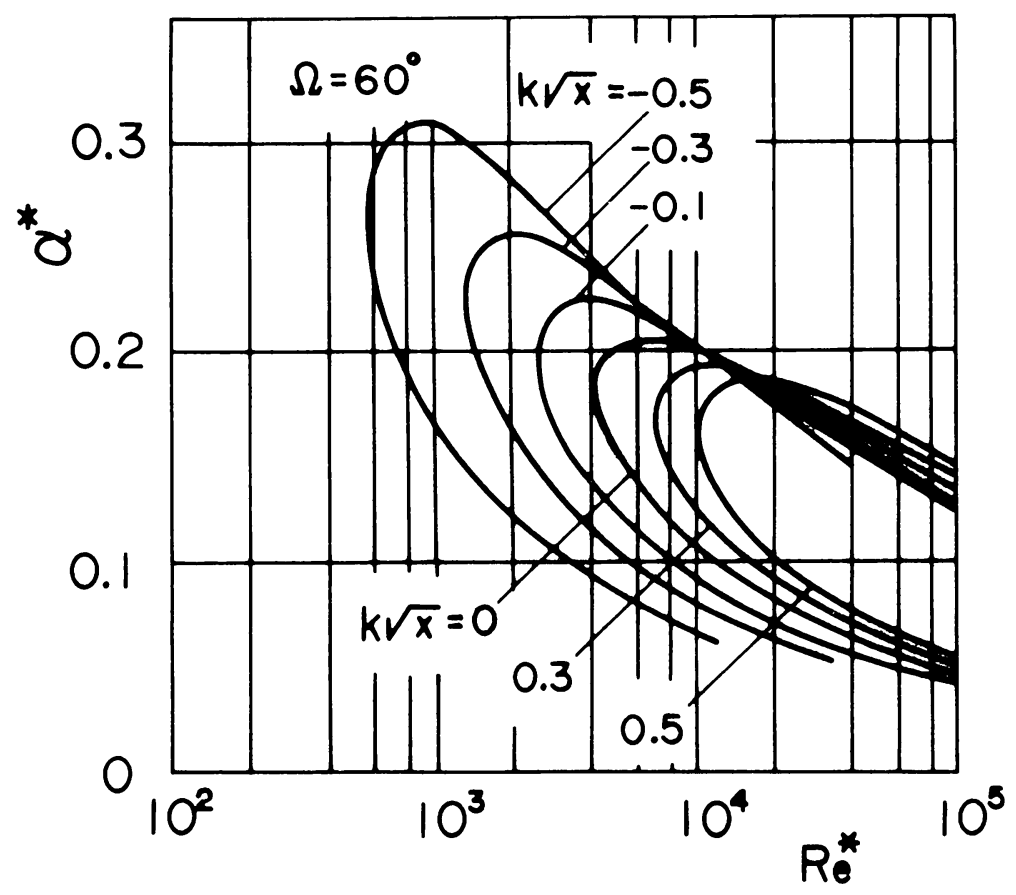

FIG. 9. Neutral stability curves $\left(\Omega=60^{\circ}\right)$.

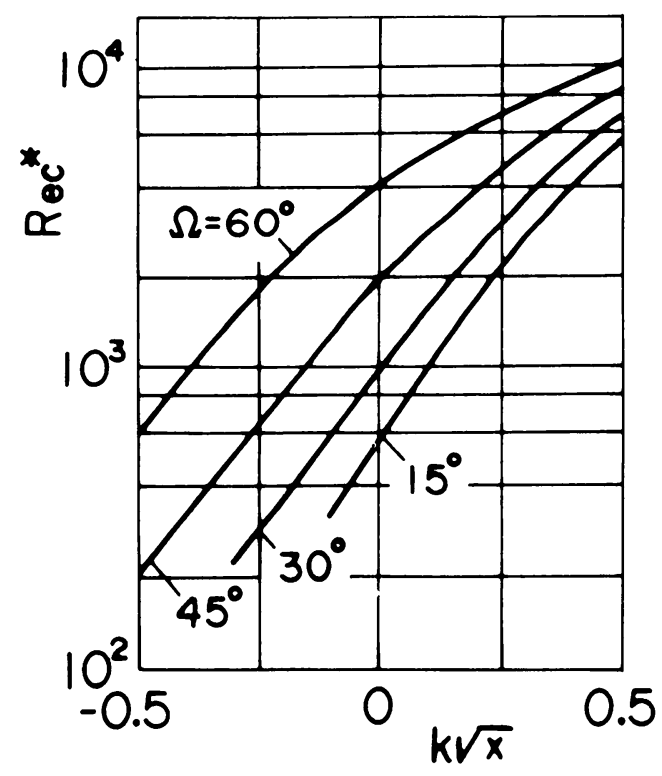

FIG. 10. Relation between the critical Reynolds number $R_{e c}^{*}$ and the suction/injection parameter $k \sqrt{x}$. 


\section{REFERENCES}

[1] C. L. Tien and I. J. Tsuji, A theoretical analysis of laminar forced flow and heat transfer about a rotating cone, Trans. ASME, J. Heat Transfer 87, 184-190 (1965)

[2] J. C. Y. Koh and J. F. Price, Nonsimilar boundary-layer heat transfer of a rotating cone in forced flow, Trans. ASME, J. Heat Transfer 89, 139-145 (1967)

[3] F. Salzberg and S. P. Kezios, Mass transfer from a rotating cone in axisymmetric flow, Trans. ASME, J. Heat Transfer 87, 469-476 (1965)

[4] Y. Furuya and I. Nakamura, Velocity profiles in the skewed boundary layers on some rotating bodies in axial flow, J. Appl. Mech. 37, 17-24 (1970)

[5] L. G. Whitehead and G. S. Canetti, The laminar boundary layer on solids of revolution, Phil. Mag. 7, 988-1000 (1950)

[6] H. Hassler, Experimentelle Untersuchungen von Langswirbeln im vorderen Staupunktgebiet eines Kreiskegels in axialsymmetrischer Anstromung, Deutsche Luft- und Raumfahrt Forschungsbericht 76, 1-49 (1976)

[7] R. Kobayashi, Instability and transition of boundary layer on a rotating cone, Trans. JSME (in Japanese) 46-B, 1900-1906 (1980)

[8] J. L. Hess and S. Faulkner, Accurate values of the exponent governing potential flow about semi-infinite cones, AIAA J. 3, 767 (1965)

[9] C. C. Lin, On the stability of two-dimensional parallel flow, Quart. Appl. Math. 3, 117-142, 218-234, 277-301 (1945/1946) 